\title{
IV. - CARACTÉRISTIQUES DE LA FRACTION GLUCIDIQUE DES ÉCHANTILLONS DE MAÏS GRAIN
}

\author{
Marie-Thérèse TOLLIER et A. GUILBOT \\ Station de Biochimie et Physico-Chimie des Céréales, I. N.R.A., \\ C. E. R.D. I. A., 91 - Massy
}

\section{RÉSUMÉ}

Les modifications de certains constituants glucidiques du maïs ont été étudiées après séchage des grains $\left(80^{\circ} \mathrm{C}\right.$ et $\left.\mathrm{I} 4 \mathrm{O}^{\circ} \mathrm{C}\right)$ soit directement après la récolte, soit après un traitement de préstockage (échauffement en cellules ou conservation par ventilation d'air refroidi). De plus, on a déterminé la sensibilité de l'amidon à l' $\alpha$-amylase bactérienne et l'activité amylasique des divers échantillons.

La température de séchage n'a pas d'effet notable sur la quantité de glucides extractibles par l'éthanol à $80^{\circ} \mathrm{GL}$. Par contre, la vitesse initiale d'amylolyse (VI) et la fraction d'amidon facilement hydrolysable $(\mathrm{FH})$ augmentent de façon sensible. L'activité $\alpha$-amylasique des grains est complètement détruite lorsque le séchage est effectué à $14^{\circ}{ }^{\circ} \mathrm{C}$.

L'échauffement en cellules avant séchage affecte fortement la composition glucidique du maïs grain. La quantité de glucides alcoolosolubles croît et des produits de dégradation de l'amidon et du raffinose peuvent être mis en évidence. Quelle que soit la température de séchage ultérieure 1'allure de 1'amylolyse des échantillons correspondant à ce traitement est semblable à celle du " témoin récolte "; seule la fraction d'amidon facilement hydrolysable est légèrement plus élevée.

La conservation par ventilation d'air refroidi avant séchage a tendance à provoquer une diminution du pourcentage de sucres de même qu'une diminution de la sensibilité de l'amidon à l'amylase bactérienne.

Pour les échantillons correspondants à ces deux traitements de préstockage, l'activité $\alpha$-amylasique est plus élevée que celle des témoins, même après séchage à température élevée ; ceci est attribué au développement des microorganismes.

\section{IN'TRODUC'TION}

Les principales connaissances concernant l'évolution biochimique du grain de maïs au cours du stockage ou du séchage ont été mentionnées dans les articles de Mon'TREUIL et al., I966; LAGRANDEUR, et al., I968. Les études suivantes sont relatives à l'évolution de la composition glucidique du maïs : glucides alcoolosolubles et amidon. Nous examinerons également l'activité amylasique des différents échantillons. 


\section{MATÉRIEL, E'T MÉTHODES}

Les travaux ont été effectués avec des lots de maïs INRA 258 ayant subi ou non divers traitements : séchage à $80^{\circ}$ ou $14^{\circ} \mathrm{C}$, soit immédiatement après la récolte $(\mathrm{R})$, soit après échauffement en cellules (E), ou après conservation par ventilation d'air refroidi $(F)$.

Sur ces différents échantillons, nous avons déterminé :

I) la composition de la fraction glucidique alcoolosoluble;

2) la teneur en amidon;

3) la sensibilité des grains d'amidon cru, à l'action de l' $\alpha$-amylase bactérienne ;

4) l'activité $\alpha$-amylasique.

Les grains de mais ont été broyés, à l'aide d'un appareil "Buhler ", en présence de neige carbonique afin d'éviter une élévation de température susceptible de provoquer des modifications chimiques ou enzymatiques des constituants du grain. La teneur en eau des échantillons a été déterminée selon la méthode de référence mentionnée dans "Méthodes analytiques des Cérétes" du CNERNA.

I) La fraction glucidique de longueur de chaîne inférieure à $\mathrm{I}_{4}$ unités glucose a été obtenue par deux extractions à ébullition avec de l'éthanol à $80^{\circ} \mathrm{GL}$ suivies de deux autres à froid. Les glucides ainsi obtenus ont été dosés par la méthode à l'anthrone de LoEwus modifiée (LoEwus, I952, Tollier, 1965) et leur identification a été effectuée par chromatographie sur papier. De plus, le glucose, le saccharose et le fructose ont été dosés séparément par des méthodes spécifiques :

- le glucose, par la méthode à la glucose-oxydase préconisée par Hugetr et Nixon, 1957 et modifiée (Tollier, I965) ; I970)

- le saccharose, par action conjuguée de l'invertase et de la glucose-oxydase (Cerning,

- le fructose par la méthode de JoHnson et al. (1964).

2) L'amidon a été dosé par la méthode d'EwERs modifiée (I965).

3) La détermination de la sensibilité de l'amidon natif à l' $\alpha$-amylase a été effectuée comme suit : une suspension à $2,5 \mathrm{p}$. Ioo de maïs broyé, en milieu tampon phosphate $\mathrm{M} / 200(\mathrm{pH}: 6,9)$ additionné de merthiolate de sodium, à I/Io ooo, est placée à $37^{\circ} \mathrm{C}$, en présence d'une préparation d' $\alpha$-amylase bactérienne (20 p. Ioo par rapport au substrat). Des prélèvements sont effectués en fonction du temps; l'hydrolyse enzymatique est bloquée en milieu alcool $80^{\circ} \mathrm{GL}$ contenant I,25 p. Ioo d'acide acétique; les glucides alcoolosolubles libérés sont dosés par la méthode à l'anthrone.

4) L'activité $\alpha$-amylasique a été déterminée selon la méthode de Perten (1966).

\section{RÉSULTATS ETT INTERPRÉTATION}

\section{I. - Glucides alcoolosolubles - Amidon}

La figure I illustre l'évolution qualitative de la composition glucidique en fonction des divers traitements.

Dans le tableau I, sont rassemblées les teneurs en glucides alcoolosolubles et en amidon des différents échantillons.

La comparaison de ces valeurs avec celles du "Témoin récolte " (TRR), c'est-àdire un maiss séché à température ambiante, permet les constatations suivantes :

- dans les maïs séchés immédiatement après la récolte $\left(R_{80}-R_{140}\right)$ la quantité de glucides alcoolosolubles est indépendante de la température de séchage. Il est 


\section{TABLEAU I}

Composition de la fraction glucidique des différents échantillons: résultats exprimés en p. 100 de matière sèche

\begin{tabular}{|c|c|c|c|c|c|}
\hline $\begin{array}{l}\text { Echan- } \\
\text { tillons }\end{array}$ & $\begin{array}{l}\text { Sucres } \\
\text { alcoolo- } \\
\text { solubles }\end{array}$ & Glucose & Saccharose & Fructose & Amidon \\
\hline $\operatorname{TR}\left({ }^{1}\right) \ldots$ & 1,75 & 0,05 & 1,01 & 0,82 & 70,8 \\
\hline R $80 \ldots$ & 1,75 & 0,12 & 0,87 & 0,66 & 71,1 \\
\hline R 140 & 1,69 & 0,06 & 0,71 & 0,62 & 70,0 \\
\hline$E_{0} \ldots \ldots$ & 4,12 & - & - & - & 68,6 \\
\hline E $80 \ldots$ & 4,22 & 1,19 & 1,29 & 1,15 & 70,0 \\
\hline E $140 \ldots$ & 4,5 & 0,99 & 1,37 & 1,24 & 70,1 \\
\hline$F_{0} \ldots \ldots$ & - & - & - & - & 70,9 \\
\hline F $80 \ldots$ & 1,41 & 0,09 & 0,79 & 0,62 & 72,0 \\
\hline F $140 \ldots$ & 1,38 & 0,06 & 0,62 & 0,56 & 70,3 \\
\hline
\end{tabular}

(1) Voir nomenclature page 610-611 (LASSERAN I)

FIG. I. - Chromatogrammes des glucides alcoolosolubles des maïs en fonction des différents traitements. $\mathrm{F}=$ fructose $; \mathrm{G}=$ glucose $; \mathrm{S}=$ saccharose $; \mathrm{M}=$ maltose $; \mathrm{Me}=$ melibiose $; \mathrm{R}=$ raffinose Solvant : $n$-butanol/acide acétique/eau $4-\mathrm{I}-5$, temps d'écoulement 4 jours.

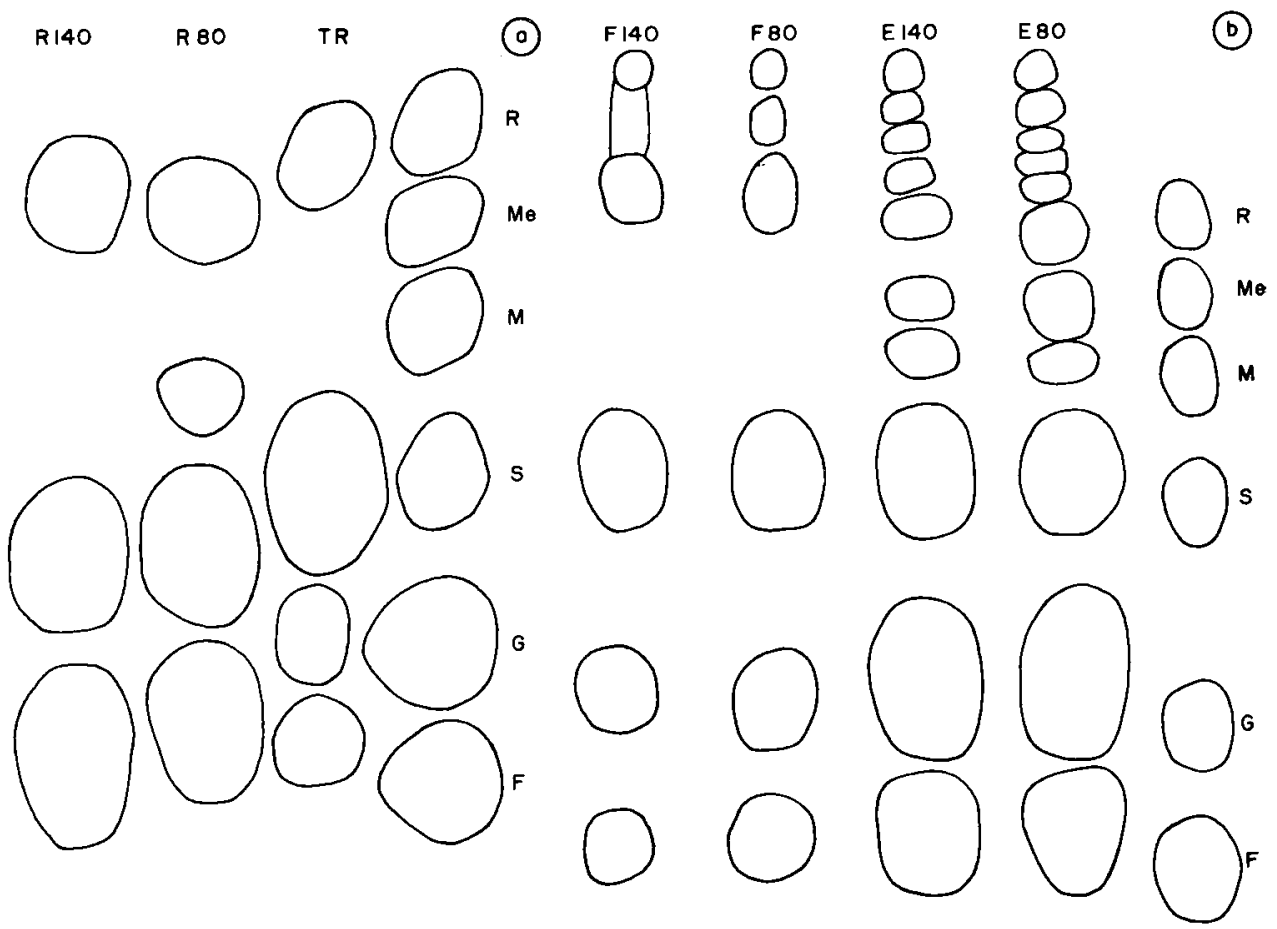


vraisemblable qu'au cours du séchage l'élévation de température est suffisamment rapide pour bloquer l'action des enzymes susceptibles de dégrader l'amidon et de fournir ainsi des oligosides de faible poids moléculaire. Par chromatographie sur papier de cette fraction (fig. I) on constate la présence des glucides habituellement trouvés dans un extrait alcoolique de mais broyé non altéré : fructose, glucose, saccharose, raffinose et de très légères traces de maltose.

- dans les maïs ayant subi un échauffement en cellules, avant d'être séchés à température ordinaire, à $80^{\circ} \mathrm{C}$ ou $\mathrm{I}_{4} \mathrm{O}^{\circ} \mathrm{C}\left(\mathrm{E}_{0}, \mathrm{E}_{80}, \mathrm{E}_{140}\right)$ une dégradation profonde commence à se manifester, se traduisant par l'augmentation très nette des glucides alcoolosolubles. La chromatographie de cette fraction montre que l'hydrolyse affecte principalement l'amidon avec libération de maltose et autres malto-oligosides; de plus, la quantité de raffinose diminue, sa dégradation se traduit par l'apparition du mélibiose et l'augmentation du fructose; enfin, la teneur en saccharose a tendance à augmenter, phénomène probablement dû à une réaction de synthèse à partir du glucose (MONTREUIL, et al., Ig66).

- dans les maïs soumis à une ventilation d'air refroidi avant séchage, $\left(\mathrm{F}_{0}\right.$, $\left.F_{80}, F_{140}\right)$ le pourcentage des glucides alcoolosolubles diminue de façon sensible. Cette diminution peut être due soit à une fermentation microbienne, développée au cours de la ventilation, soit à une respiration du grain, soit à la conjugaison de ces deux phénomènes. Dans ce dernier cas, il n'est pas possible de déceler des glucides provenant de l'hydrolyse de l'amidon et la teneur en saccharose semble diminuer légèrement par rapport à celle des lots $R$. ficative.

$\mathrm{L}_{\mathrm{L}}$ teneur en amidon de tous les échantillons étudiés ne varie pas de façon signi-

\section{2. - Sensibilité de l'amidon à l'a-amylase}

Les résultats concernant la sensibilité de l'amidon à l' $\alpha$-amylase bactérienne sont illustrés par les courbes des figures $2,3,4$. Pour mieux préciser les phénomènes observés en fonction des différents traitements, nous avons suivi l'évolution de la

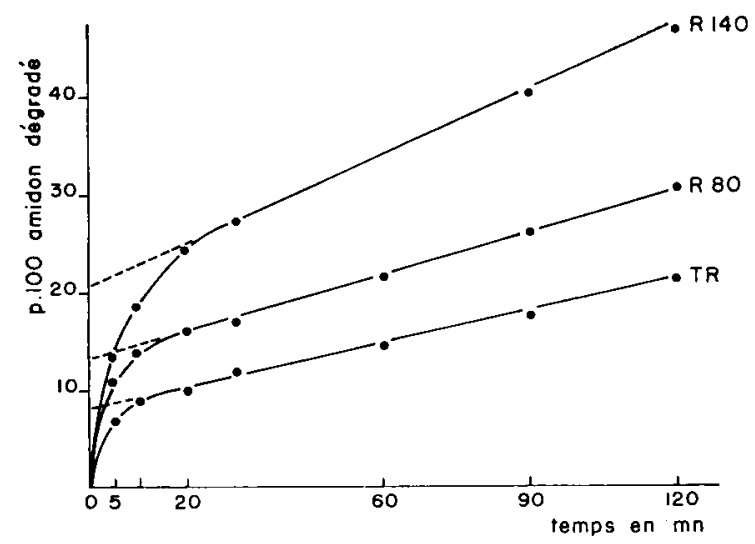

FIG. 2. - Evolution, en fonction du temps, de l' $\alpha$-amylolyse, à l'état cru, des maïs "Témoin récolte " $(T . R) \mathrm{R}_{\mathbf{8 0}}$ et $\mathrm{R}_{\mathbf{1 4 0}}$

vitesse initiale (VI) et finale (VF) d'hydrolyse et de la quantité de fraction facilement hydrolysable (FH) (tabl. 2). 
TABLEAU 2

Caractéristiques des courbes d' $\alpha$-amylolyse

\begin{tabular}{|c|c|c|c|}
\hline Echantillon & $\mathrm{VI}(\mathbf{I})$ & $\operatorname{VF}\left({ }^{2}\right)$ & $\mathrm{FH}\left({ }^{3}\right)$ \\
\hline TR & 7,2 & 0,60 & 8,0 \\
\hline$R_{80}$ & 11,0 & 0,62 & 13,0 \\
\hline$R_{140}$ & 13,5 & 1,0 & 20,75 \\
\hline $\mathrm{E}_{0}$ & 7,6 & 0,7 & 11,25 \\
\hline$E_{80}$ & 7,2 & 0,72 & 10,0 \\
\hline$E_{140}$ & 6,0 & 0,72 & 10,0 \\
\hline$F_{0}$ & 10,0 & 1,0 & 12,5 \\
\hline $\mathrm{F}_{80}$ & 4,2 & 0,6 & 6,8 \\
\hline $\mathrm{F}_{140}$ & 7,3 & 1,04 & 8,75 \\
\hline
\end{tabular}

(1) VI : Vitesse initiale d'amylolyse, exprimée en pourcentage d'amidon dégradé en 5 minutes.

(2) VF : Vitesse finale d'amylolyse, exprimée en pourcentage d'amidon dégradé en moyenne en 5 minutes pendant la 1 ere heure.

( $\left.{ }^{3}\right)$ FH : Fraction facilement hydrolysable, déterminée graphiquement par extrapolation de la partie linéaire des courbes d'amylolyse.

- Chez les maïs séchés à température ambiante, à $80^{\circ}$ ou $140^{\circ} \mathrm{C}$, après récolte (fig. 3), la valeur des paramètres mesurés VI, VF et $\mathrm{FH}$ présente une augmentation importante après le séchage, augmentation d'autant plus marquée que la température est plus élevée $\left(R_{80}\right.$ et $\left.R_{140}\right)$.

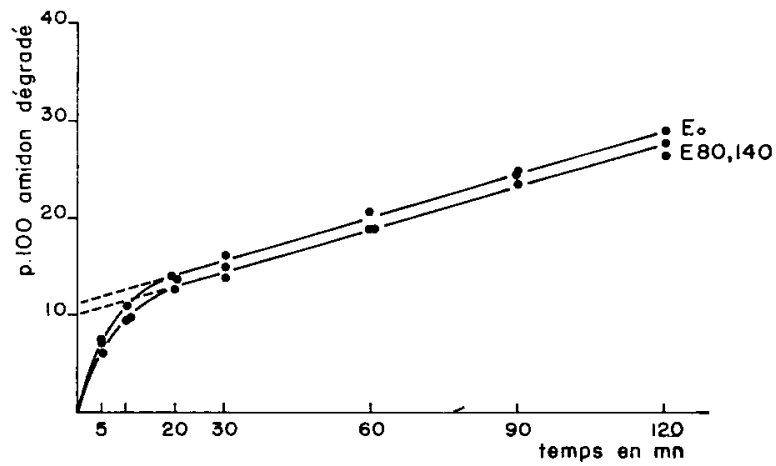

FIG. 3. - Évolution, en fonction du temps de l'a-amylolyse, à l'état cru, des maïs séchés après êchauffement $E_{00}, E_{80}$ et $E_{140}$ 
- Chez les maìs ayant subi un échauffement avant d'être séchés, à diverses températures (température ambiante, 80 ou $\mathrm{r}_{4} \mathrm{0}^{\circ} \mathrm{C}$ ) l'allure de l'amylolyse reste la même, comme si à la suite de ce préstockage, 1'amidon était devenu moins sensible aux altérations dues au traitement thermique ultérieur; les valeurs de VI et VF sont peu différentes de celles du " témoin récolte", par contre, la quantité de fraction facilement hydrolysable ( $\mathrm{FH}$ ) est légèrement plus élevée (fig. 4); ce qui laisserait entendre que les modifications observées sur les échantillons précédents portaient sur la fraction d'amidon qui a été hydrolysée au cours du préstockage.

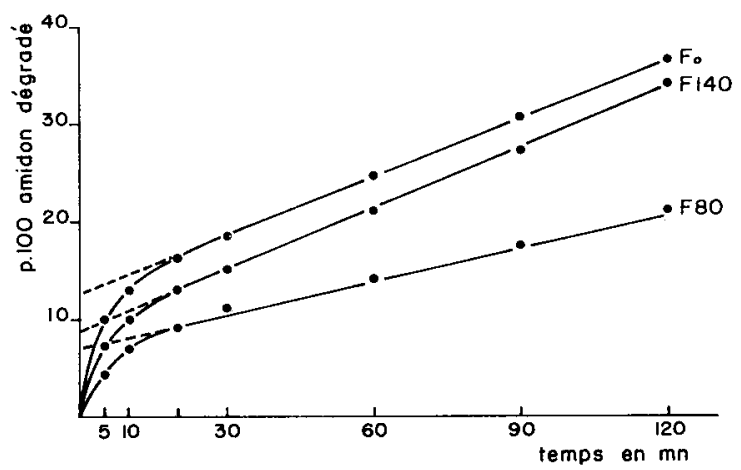

Fig. 4. - Évolution, en fonction du temps, de l' $\alpha$-amylolyse, à l'état cru, des maïs séchés après ventilation réfrigérée

$$
\mathrm{F}_{0}, \mathrm{~F}_{80} \text { et } \mathrm{F}_{140}
$$

- Chez le maïs soumis à une ventilation d'air refroidi avant séchage, $\left(F_{0}\right)$, $\mathrm{VI}, \mathrm{VF}$ et $\mathrm{FH}$ ont augmenté par rapport à ('TR) ; on observe, de plus, que l'action de la chaleur après ce traitement a pour effet de diminuer la quantité de fraction facilement hydrolysable, de même que VI et VF, en particulier pour $\mathrm{F}_{\mathbf{8 0}}$.

\section{3. - Activité $\alpha$-amylasique}

Les déterminations de l'activité $\alpha$-amylasique (tabl. 3), exprimée en unités A par gramme de substance, permettent de constater qu'après séchage à $80^{\circ} \mathrm{C}$, 1'activité des amylases du grain est déjà fortement diminuée et, à $I 40^{\circ} \mathrm{C}$, elle devient pratiquement nulle. Par contre, après les traitements d'échauffement et de conservation

TABLEAU 3

Activité $\alpha$-amylasique en unité $A$

(ICC. standardisée Perten, I966)

\begin{tabular}{l|c}
\hline TR & 1,25 \\
R 80 & 0,7 \\
R 140 & 0,05 \\
$E_{0}$ & 30,0 \\
E 80 & 10,0 \\
E 140 & 3,3 \\
$F_{0}$ & 4,5 \\
F 80 & 2,4 \\
F 140 & 0,24 \\
\hline
\end{tabular}


par ventilation d'air refroidi, suivis d'un séchage à température normale, on observe une brusque augmentation de cette activité, augmentation plus importante dans le premier cas que dans le second. Ceci peut être attribué soit à un début de germination, soit au développement de micro-organismes, soit aux deux phénomènes.

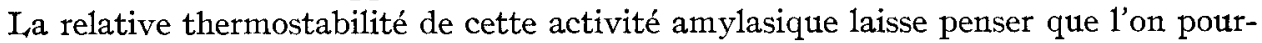
rait avoir, au moins pour une part, des amylases d'origine bactérienne.

\section{CONCLUSIONS}

Des trois traitements mis en œuvre, "l'échauffement en cellules " est celui qui semble avoir dégradé le plus l'amidon. Par rapport au " témoin récolte", l'augmentation de l'activité amylasique propre au grain ou provenant des micro-organismes qui se sont développés, au cours du prétraitement est responsable de l'accroissement des glucides de faible poids moléculaire. On peut noter également qu'après ce préstockage, le traitement thermique a un effet nettement moins important sur la sensibilisation de l'amidon à l'amylase que lorsqu'il est effectué directement après récolte et ceci quelle que soit la température. En ce qui concerne l'activité amylasique, elle est encore importante même après le séchage à $140^{\circ} \mathrm{C}$.

La conservation par ventilation d'air refroidi des maïs avant séchage a pour conséquence de diminuer la quantité de glucides alcoolosolubles, ceci étant probablement dû à une certaine activité métabolique du grain qui subsiste à basse température. La sensibilité à l' $\alpha$-amylolyse est également affectée ; plus importante après un séchage à température ordinaire, elle tend à diminuer à la suite de séchage à températures plus élevées. Quant à l'activité amylasique, elle est encore décelable en quantité non négligeable.

\section{SUMMARY}

\section{IV. - CHARACTERISTICS OF THE CARBOHYDRATE COMPOSITION OF THE MAIZE KERNEI,}

The change of certain sugars in the maize kernel was studied after drying ( 80 and $140^{\circ} \mathrm{C}$ ) and during storage of wet corn (spontaneous heating in bin and cooling ventilation). Alphaamylolysis of starch and alpha-amylase activity were also determined on various samples.

The drying temperatures have no effect on the percentage of alcohol-soluble sugars. However the initial speed of amylolysis (VI) and the easily hydrolysable starch fraction (FH) increase appreciably. The alpha-amylase activity is completely destroyed by drying at $140^{\circ} \mathrm{C}$.

The in-bin spontaneous heating before drying deteriorates strongly the sugar constituants of maize. The alcohol-soluble carbohydrates increase and it is possible to detect degradation products of starch and raffinose by paper chromatography. The action pattern of alpha-amylolysis of maize heated in cells before drying remains essentially comparable to the control samples, and this independently of the temperature of ulterior drying.

The treatment by cooling ventilation before drying decreases the amount of carbohydrates and the susceptibility of starch to bacterial amylase.

In all samples stored before drying, alpha-amylase activity is more important than in the control samples, even after high-temperature drying; this is attributed to the enzymes elaborated by micro-organisms. 


\section{RÉFÉRENCES BIBLIOGRAPHIQUES}

Cerning J., I97o. Contribution à l'étude de l'évolution de la composition glucidique des grains de céréales au cours de la maturation : maïs, blé, orge. Thèse de Doctorat d'Université, Lille.

Huggett A. S., Nixon D. A., I957. Enzymic determination of blood glucose. Biochem J., 66, I2 p.

EWERS E., I965. Determination of starch by extraction and dispersion with hydrochloric acid. International Organization for Standardization (ISO/TC 93/WGL).

Johnson G., Lambert C., Johnson D. K., Sunderwirth S. G., I964. Colorimetric determination of glucose, fructose and sucrose in plant material using a combination of enzymatic and chemical methods. J. Agric. Food Chem., 12, 216-219.

CAHIERS TEChNiQUES DU CNERNA, I958. Les méthodes analytiques des Céréales. 111, I7I-I86.

Lagrandeur G., Tollier M.-Th., Guilbot A. 1968. Evolution de certains constituants du grain de maïs en fonction de diverses conditions de stockage ou de séchage. Inds. Alim. agr., 85, 837-842.

Loewus F. A., 1952. Improvement in anthrone method for determination of carbohydrates. Anal. Chem., 24, 219 .

Montreuil J., Monsigny M., Leclerco-Ducastel G., Mercier Ch., Tollier M. T., Guilbot A., I966. Influence des conditions de production, de récolte et de stockage sur la composition biochimique des céréales; répercussions des variations sur la valeur nutritionnelle : II. glucides. Ann. Nutr. Alim., 20, I65-I75.

Perten H., I966. A colorimetric method for the determination of $\alpha$-amylase activity. Cereal Chem., 43, 336-342.

Tollier M. Th., I965. Contribution à l'étude du rayonnement $\lambda$ sur les caractères physicochimiques de l'amidon et sa sensibilité aux amylases. Thèse d'Ingénieur du C. N. A. M, Paris. 